\title{
CINCO AÑOS DE IMPLEMENTACIÓN DEL TRATADO DE LIBRE COMERCIO ENTRE COLOMBIA Y ESTADOS UNIDOS: ANÁLISIS DEL DESARROLLO SOBRE EL SECTOR PRODUCTOR ARROCERO
}

\author{
Anderson Javier Quintero ${ }^{1 *}$; Scarlet Yamely Sotomayor Tacuri2 \\ ${ }^{1}$ Consultor del Consejo Noruego para Refugiados, Bogotá-Colombia. \\ ${ }^{2}$ Corporación Universitaria Minuto de Dios, Bogotá-Colombia \\ *Autor para correspondencia: e-mail: scarletsotomayor@otmail.com \\ Recibido: 2018/03/06 \\ Aprobado: 2018/06/12 \\ D0I: https://doi.org/10.26621/XIV18.2018.06.A01.PUCESI.2550.6684
}

\section{RESUMEN}

Este trabajo surge de una tesis de maestría ${ }^{1}$ cuyo objetivo fue, analizar las implicaciones económico -sociales que han tenido el Tratado de Libre Comercio entre Colombia y Estados Unidos sobre el sector productor arrocero en el Departamento del Meta. Se abordaron dos categorías de análisis: i) Uso de variables macroeconómicas: Ventaja Comparativa Revelada -ICVR-, Índice de Intensidad Importadora -III- e Índice de Complementariedad Comercial -ICC- para identificar el flujo comercial entre ambos países; ii) Mecanismos de lucha social por las condiciones del mercado arrocero. Se encontró que, en términos económicos, Colombia tiene un déficit comercial en la producción arrocera a causa de las malas condiciones de intercambio. En términos sociales, afectan de forma negativa las condiciones de vida de los pequeños agricultores y aumenta el despojo territorial.

Palabras claves: Tratado de Libre Comercio, poder, desarrollo económico, crecimiento económico, movimiento social campesino, despojo

\begin{abstract}
This work arises from a master's thesis ${ }^{1}$, whose objective was to analyze the economic and social implications of the Free Trade Agreement between Colombia and the United States on the rice producing sector in the Department of Meta. Two categories of analysis were addressed: i) Use of macroeconomic variables: Revealed Comparative Advantage -ICVR-, Importing Intensity Rate and Commercial Complementarity Rate to identify the commercial flow between both countries; ii) Mechanisms of social struggle for the conditions of the rice market. It was found that, in economic terms, Colombia has a trade deficit in rice production due to poor exchange conditions. In social terms, they negatively affect the living conditions of small farmers and increase territorial dispossession.
\end{abstract}

Keywords: Free Trade Agreement, power, economic development, economic growth, peasant social movement, dispossession 


\section{INTRODUCCIÓN}

La noción de crecimiento, como mecanismo de desarrollo, surge de las vertientes clásicas de la economía con Adam Smith (1776), Alfred Marshall (1890), Robert Solow (1956), Lewis (1954) y Leontief (1985). Sus aportes son importantes para el concepto de crecimiento micro y macroeconómico, permitiendo articular la economía local con la global. Esta idea se fundamentó en la acumulación de capital e incremento de plataformas financieras internacionales (Lewis, 1954) bajo la aparición de brechas de desigualdad económica de países ricos y pobres (Leontief, 1985), cuyo desarrollo está cimentado en la libre competencia y el libre mercado.

Durante las tres últimas décadas, el Acuerdo General sobre Aranceles Aduaneros y Comercio -GATTdejó de ser un acuerdo entre Estados miembros para ser un acuerdo internacional (Cox, 2002, p. 81). La Organización Mundial del Comercio -OMC- modificó el comercio de las materias primas, manufacturas y la inversión extranjera directa (aspectos que terminaron siendo liberalizados y regulados por el orden mundial); el abandono del patrón oro ${ }^{2}$ y la implementación del dólar como moneda de cambio (Keohane, Nye y Stiglitz, 1977). El Tratado de Libre Comercio, conocido como mecanismo de integración comercial entre dos o más países, con el fin de crear una zona de libres de aranceles, fue propuesto por Bill Clinton en diciembre de 1994 (Acosta, 2006) como alternativa a lo presentado por la OMC.

Según la OMC, a partir de 1984 hasta 25 de enero de 2018, se han suscrito más de 455 acuerdos comerciales, de los cuales 284 están siendo modificados (OMC, 2018). Ante esta inclusión de los países a la cadena de valor internacional, OXFAM sostiene que "el $82 \%$ de la riqueza generada fue a parar a manos del $1 \%$ más rico; mientras el 50\% más pobre de la población mundial obtuvo el 0\%" (OXFAM, 2018). Por ende, tener un libre mercado (sin irregularidades) no implica llegar a un desarrollo social.

EI TLC entre Colombia y Estados Unidos fue firmado el 14 de mayo de 2012. Cinco años después de la implementación es necesario un análisis, desde la economía política internacional del desarrollo, para mostrar el impacto en los pequeños productores arroceros. El fin es desmitificar el TLC sobre este sector y su contraste con la noción de desarrollo económico. El documento está compuesto por tres núcleos de trabajo: i) El aspecto económico del sector productor arrocero, desde las variables macroeconómicas de inversión; ii) el ámbito social de la comercialización de los pequeños productores arroceros, su movilización y la construcción de la Mesa Agraria Nacional; iii) conclusiones sobre una construcción propia de desarrollo.

\section{MATERIALES Y METODOLOGÍA}

El objetivo del trabajo fue analizar las condiciones económicas y sociales de los productores arroceros del Departamento del Meta, desde la economía política internacional, durante los cinco años de la implementación del acuerdo. Para ello, se utilizaron dos métodos: el análisis documental de textos académicos, prensa digital, estudios económicos, bases de datos del Departamento Nacional de Planeación (DNP), del Departamento Administrativo Nacional de Estadística (DANE) y Sistema de Información sobre Comercio Exterior -Sicex-. Y la observación participante con instrumentos como entrevistas semiestructuradas a las organizaciones sociales de base (MUCAPOC, FENSUAGRO, AGROLLANOS, AGAMETA, DIGNIDAD ARROCERA) del departamento.

\section{RESULTADOS Y DISCUSIÓN}

\section{Análisis de la apertura económica y su implicación en el mercado arrocero colombiano}

El proceso de apertura económica en Colombia inició en los años 1990, con la liberalización del mercado financiero por parte del presidente César Gaviria (1990-1994). Ernesto Samper (1994-1998) apoyó el Área de Libre Comercio de las Américas (ALCA) ${ }^{3}$, Andrés Pastrana (1998-2002) continuó con la puesta en marcha del Plan Colombia4 ${ }^{4}$, mientras que Álvaro Uribe (2002-2010) inició la firma del TLC con Estados Unidos. Por último, Juan Manuel Santos (2010-2018) ha rubricado la suscripción de los TLC con Estados Unidos y la Unión Europea (Figura 1). Su resultado fue la apertura completa hacia el mercado internacional. 
Figura 1. Proceso de apertura comercial entre Colombia y Estados Unidos

\section{Proceso de Apertura Comercial entre Colombia y} Estados Unidos

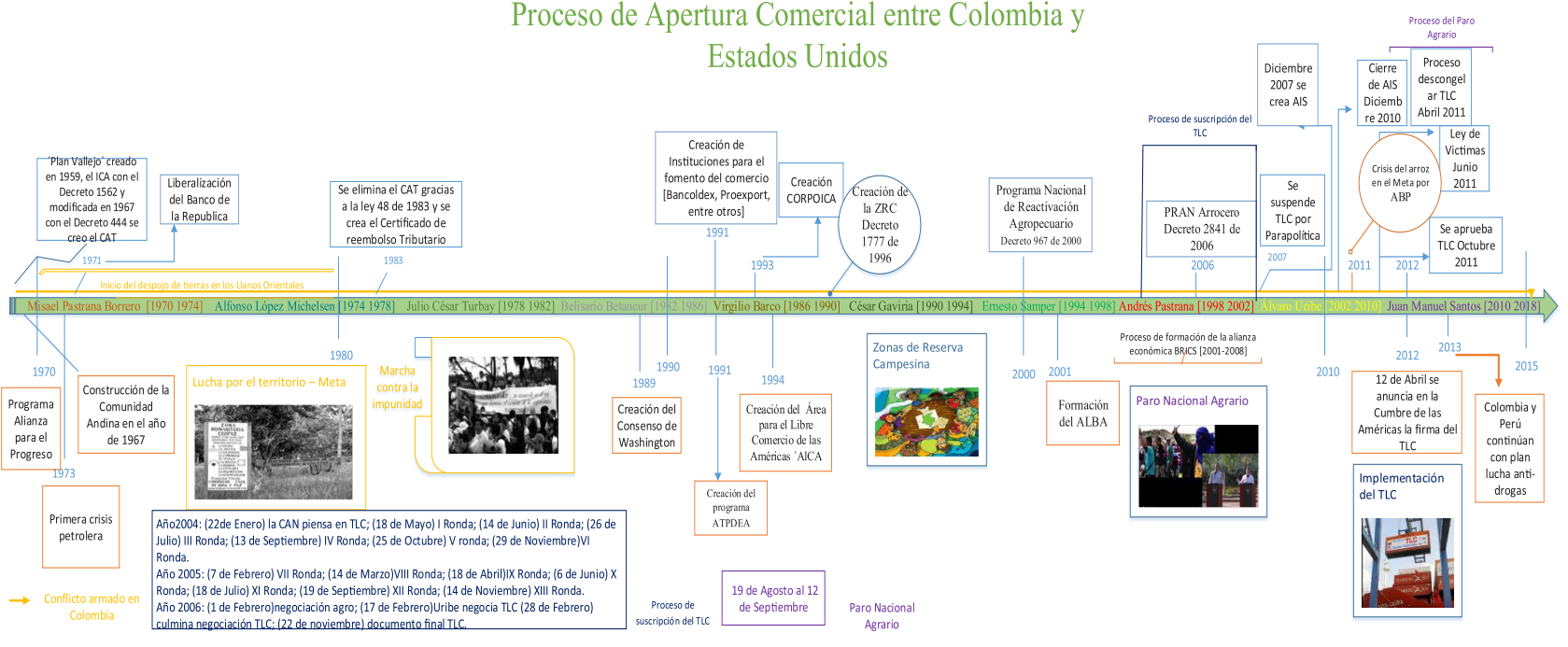

Fuente: Elaboración propia a partir de la información de documentos del CINEP (2014), Organización de Estados Americanos (2014), Ministerio de Agricultura (2011), Ministerio de industria y turismo (2014) y Procolombia (2012).

Desde la suscripción del TLC en Colombia, se vive un proceso de discontinuidad de normativas internas restrictivas, disminución de aranceles y liberalización progresiva del mercado local, en los aspectos institucionales del acuerdo, así como los compromisos de transparencia para la resolución de controversias; el acceso a productos agropecuarios como normas de origen, procesos aduaneros; $y$ temas sobre propiedad intelectual, laborales y ambientales.

Basados en los principios de la teoría económica de David Ricardo sobre la ventaja comparativa ${ }^{5}$ (1817), los autores MacDougall (1951), Stern (1962), Ballassa (1965), Vollrath (1991), Scott y Vollrath (1992) la probaron con éxito, convirtiéndola en fuente de medición para el intercambio comercial entre países. De esa forma, la producción colombiana de arroz se medirá por ventaja comparativa relativa (o índice de ventaja comparativa relativa) y determinar el flujo comercial de mercancías entre países, mediante sus costos relativos (Balassa, 1965). Para ello, se usarán el Índice de Ventaja Comparativa Revelada (ICVR), El Índice de Intensidad Importadora (III) y el Índice de Complementariedad Comercial (ICC) (Tabla 1). Las variables obtenidas se compararán con la base de datos del Departamento Nacional de Planeación (DNP).

Tabla 1. Variables macroecómicas

\begin{tabular}{|c|c|c|}
\hline $\begin{array}{c}\text { Índice de Ventaja Comparativa } \\
\text { Revelada (ICVR) }\end{array}$ & Adice de Intensidad Importadora (III) & $\begin{array}{l}\text { Índice de Complementariedad } \\
\text { Comercial (ICC) }\end{array}$ \\
\hline $\begin{array}{l}\quad \mathbf{I V C R}_{\mathrm{ij}}=\left(\mathrm{X}_{\mathrm{ij}} / \mathrm{X}_{\mathrm{i}}\right) /\left(\mathrm{X}_{\mathrm{wj}} / \mathrm{X}_{\mathrm{w}}\right) \text { (1) } \\
\mathbf{I V C R}_{\mathrm{ij}} \text { Índice de Ventaja Comparativa } \\
\text { Revelada del país } \mathbf{i} \text { para el producto } \mathbf{j} \\
\mathbf{X}_{\mathbf{i j}}: \text { Valor de las exportaciones del } \\
\text { país } \mathbf{i} \text { del producto } \mathbf{j} \\
\mathbf{X}_{\mathbf{i}} \text { : Valor de las exportaciones totales } \\
\text { del país } \mathbf{i} \\
\mathbf{X}_{\mathrm{wj}} \text { : Valor de las exportaciones mun- } \\
\text { diales del producto } \mathbf{j} \\
\mathbf{X}_{\mathbf{w}} \text { : Valor de las exportaciones totales } \\
\text { mundiales }\end{array}$ & 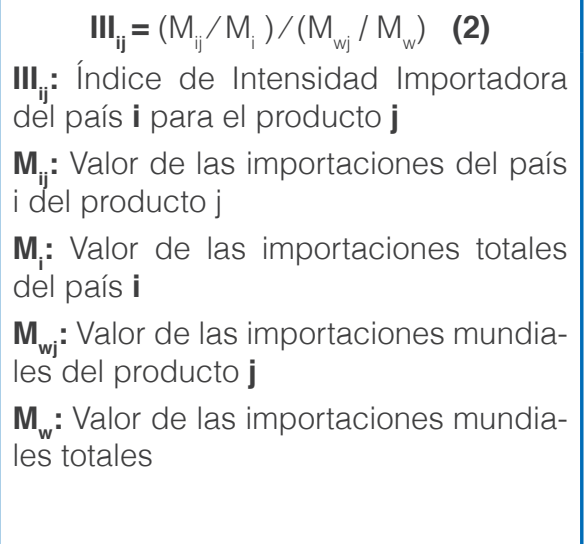 & 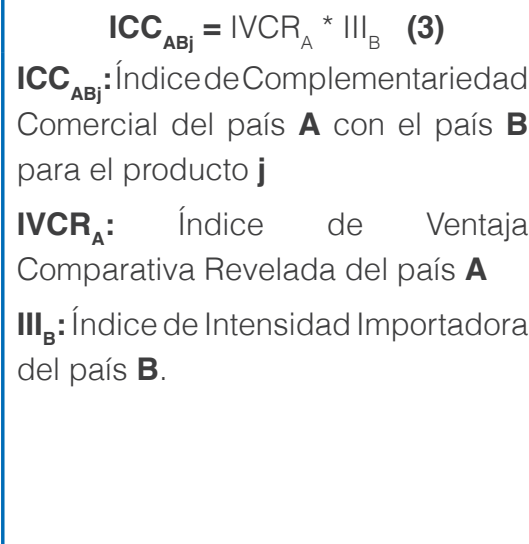 \\
\hline
\end{tabular}

Fuente: DNP, 2017

${ }^{5}$ Sostiene que un país puede comercializar eficientemente un producto que tenga alguna ventaja de producción [insumos, capital humano] y puede existir comercio entre dos países, si uno de ellos tiene una desventaja absoluta en la elaboración de sus productos. 
El IVCR representa el nivel de participación en términos comerciales del arroz (producto i) entre Colombia (país j) y Estados Unidos. Si el valor es >1, el arroz tendrá una ventaja comparativa en el mercado estadounidense. EI ICC resulta de la intensidad importadora entre los socios comerciales con respecto al arroz (producto j), Colombia (país i) y Estados Unidos. Estos valores son cuantifícales de 0 en adelante, siendo > 1 la especialización en exportación que tiene un país del arroz (producto j) sobre el otro. Finalmente, está el III que muestra en términos relativos la especialización de importación del arroz (producto j), si este índice es > 1 entre Estados Unidos (país A) y Colombia (país B) quiere decir que tienen complementariedad comercial; de lo contrario no.

Por tanto, los resultados evidencian que el IVCR entre Colombia y Estados Unidos es desigual, los valores indican que la relación es de -0.979 a -1.000 desde el 2010 hasta el 2015 en el arroz. Respecto al III en 2015, es de 0.319 que es la sumatoria de todos los Índices de Complementariedad Comercial y, en este caso, son de 0.000. Es decir, las exportaciones del arroz de Colombia a Estados Unidos no representan una gran cantidad para el comercio mundial; por el contrario, Colombia se ve altamente afectada en términos de reciprocidad comercial.

Figura 2. Balanza comercial entre Colombia y Estados Unidos

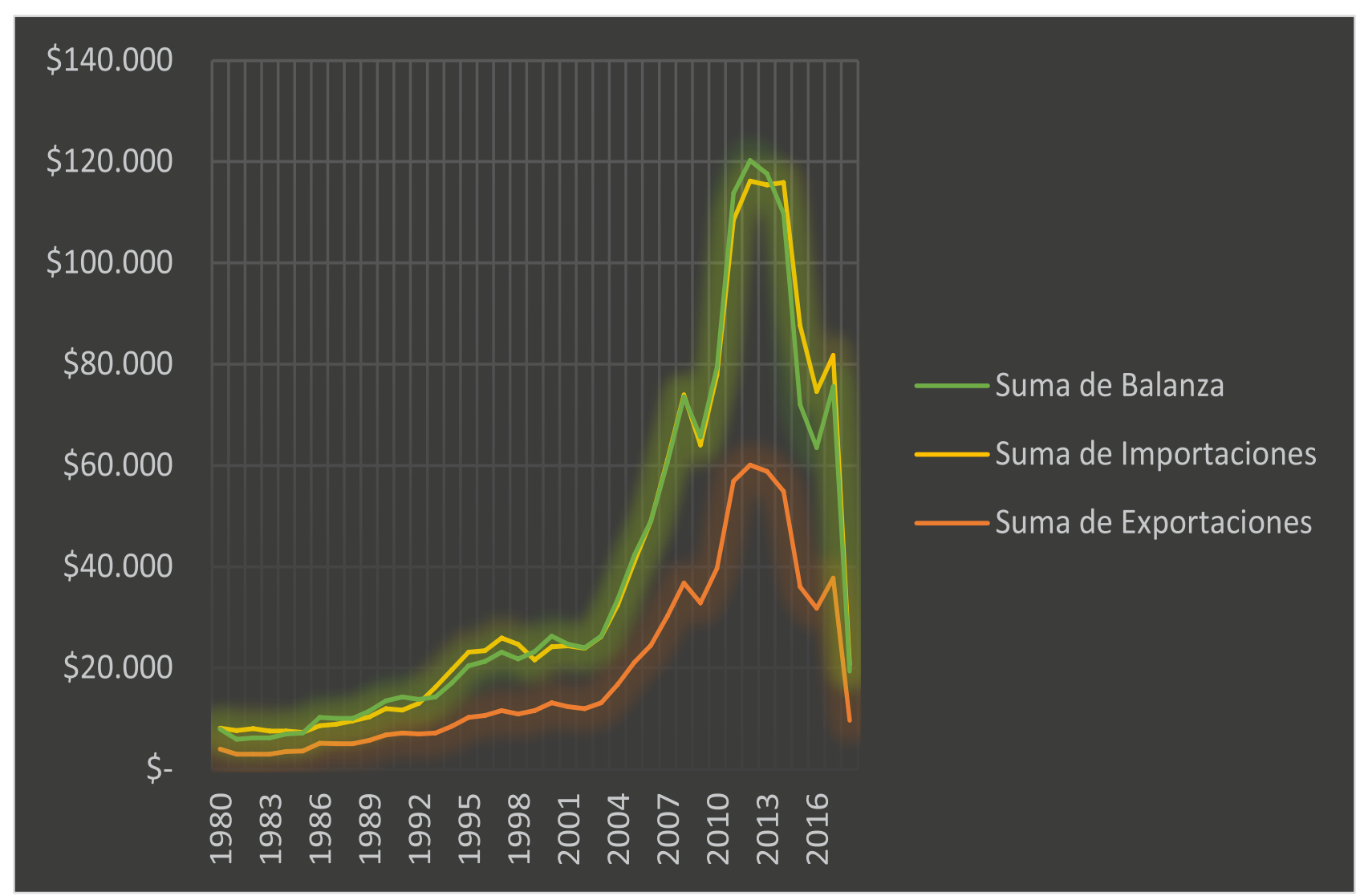

Fuente: Compilación de información del DANE (Balanza Comercial) y DNP (Análisis del comercio internacional colombiano y diversificación de exportaciones (2002-2015): Canadá, Estados Unidos y Unión Europea). Recuperado de https://www.dane.gov.co/ index.php/estadisticas-por-tema/comercio-internacional/balanza-comercial.

Según estas cifras económicas (Figura 2) se evidencia que la balanza comercial entre Colombia y Estados Unidos fue deficitaria en 2006, 2007, 2014 y 2015 por valor de USD \$143 millones, USD \$824 millones, USD \$6.292 millones y USD \$15.907 millones respectivamente, debido al aumento de las importaciones y descenso de las exportaciones (DNP, 2017). Otro indicador importante es el nivel de apertura económica que, en relación con las exportaciones, pasó del 14,3\% en el 2004 a 12,2\% en el 2015 (DNP, 2017). Al 
final, la economía local colombiana importa más de lo que exporta al mercado estadounidense. El libre mercado especializa un solo sector y desestimula a otros gremios, generando una desventaja comparativa de intercambio (Krugman, 2000).

\section{Mecanismos de participación social de los arroceros colombianos frente a la apertura económica}

Las condiciones comerciales del arroz en Colombia no han mostrado el efecto esperado. La institucionalidad "se encargó de crear un corporativismo que minimizó la acción del mismo, reflejando el dominio del sector orientado a la economía mundial sobre el nacional” (Cox, 2013, p.24). En ese sentido, este apartado muestra las diferentes posiciones de los arroceros frente a las afirmaciones del gobierno nacional sobre el TLC.Las organizaciones arroceras en el Departamento del Meta, quienes evaluaron que cuando se negoció el TLC no se tuvo en cuenta: primero, la revaluación de las monedas que afectan el mercado comercial internacional (se encarecen las exportaciones y se abaratan las importaciones); segundo, la quiebra de los agricultores, el capital público y privado no financian sectores de alto riesgo como el agro; tercero, el desplazamiento del mercado financiero afecta el desarrollo, genera déficit en las cuentas corrientes del país (cuentas internas), lo que se traduce en un trastorno financiero y endeudamiento en las cuentas externas (Canal Llanero, 2015).

Así mismo, el Capítulo 2 del acuerdo comercial estableció tres compromisos importantes: el primero, las solicitudes colombianas en términos sanitarios y fitosanitarios serían resueltas por organizaciones estadounidenses; segundo, las evaluaciones técnicas de riesgo sobre productos agrícolas para potenciar la industria arrocera; tercero, mantener la salvaguardia (arancel) durante 19 años para proteger el sector arrocero (Fedesarrollo, 2007).

Otra estrategia del gobierno fue la creación del Programa Nacional de Reactivación Agropecuario -PRAN (Arrocero), implementado mediante el Decreto 2841 de 2006 y Fondo de Solidaridad Agropecuario -FONSAcon la administración de FINAGRO (Finagro, 2015). A pesar de sus buenas intenciones, estos programas no han tenido el impacto esperado porque cuando sube el precio del arroz, sube por las importaciones, el contrabando, los altos costos en los insumos y los combustibles; por carencia de investigación, asistencia técnica convertida en venta de insumos, competencia con unos arroces subsidiados, malas condiciones de comercialización y la devaluación del dólar. No tenemos arroz porque lo desestimularon. Y no se cuenta con una seguridad alimentaria, porque no se ha promovido la soberanía alimentaria (entrevista \#5, 21 de febrero 2015).

Ante este panorama, no es posible pensar en una soberanía alimentaria, definida como una producción agrícola autosostenible, amigable con el medio ambiente y producto de un proyecto de vida campesino comunitario (CINEP, 2014), porque las zonas donde se produce más arroz (la Región Central, Llanos, Costa Norte, Santander, Bajo Cauca) tienen un oligopolio empresarial de molienda [Arroz Diana, Arroz Roa, Arroz Flor Huila], que regulan indiscriminadamente el precio con el que los pequeños productores que, ocupan más del 70\% del total, dejan el campo y se convierten en jornaleros, cocineras, maquinistas, regadores, administrador y dueño del cultivo (Figura 3) (Fedearroz, 2011, p.127).

Frente a esta problemática, los movimientos campesinos buscaron mitigar los efectos de la inclusión de productos agrícolas al mercado internacional con el Decreto 1777 de 1996, sobre Zonas de Reserva Campesinas (ZRC) . Pero, esta iniciativa tiene dos problemas que le han significado al campesino la pérdida de tierra cultivable y productiva, las Zonas de Interés de Desarrollo Rural Económico y Social -ZIDRES(Proyecto de Ley 133 de 2014) que cambiaría los espacios de autonomía campesina a zonas de desarrollo industrial a pequeña escala; y el desplazamiento de la población por la amenaza de grupos armados del Bloque Centauros, los Masetos, Autodefensas Campesinas del Casanare (paramilitares) y el Ejército de Liberación Nacional (guerrillero). 
Figura 3. Departamentos arroceros en Colombia

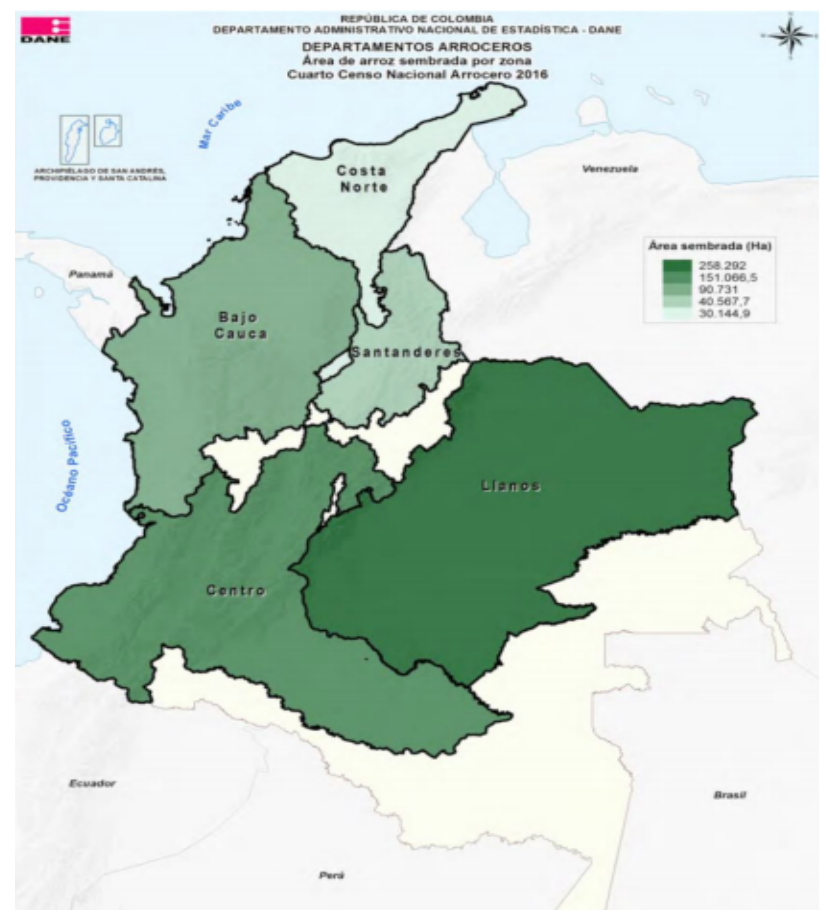

\begin{tabular}{|c|c|c|c|}
\hline \multirow{2}{*}{$\begin{array}{c}\text { ZONA } \\
\text { ARROCERA }\end{array}$} & \multirow{2}{*}{ DEPARTAMENTO } & \multicolumn{2}{|c|}{ ÁREA SEMBRADA AÑO } \\
\hline & & $\mathrm{Ha}$. & \\
\hline \multirow{3}{*}{ BAJO CAUCA } & $\begin{array}{l}\text { ANTIOQUIA } \\
\text { BOLIVAR }\end{array}$ & & \\
\hline & $\mathrm{CHOCO}$ & 90.751 & $15,9 \%$ \\
\hline & CORDOBA & & \\
\hline \multirow{7}{*}{ CENTRO } & SUCRE & & \\
\hline & CAQUETA & & \\
\hline & $\begin{array}{l}\text { CAUCA } \\
\text { CUNDINAMARCA }\end{array}$ & & \\
\hline & HUILA & 151.067 & $26,5 \%$ \\
\hline & NARIÑO & & \\
\hline & TOLIMA & & \\
\hline & VALLE DEL CAUCA & & \\
\hline \multirow{5}{*}{ COSTA NORTE } & YONDÓ (ANTIOQUIA) & & \\
\hline & ATLANTICO & & \\
\hline & CESAR & 30.125 & $5,3 \%$ \\
\hline & LA GUAJIRA & & \\
\hline & MAGDALENA & & \\
\hline \multirow{5}{*}{ LLANOS } & ARAUCA & & \\
\hline & CASANARE & & \\
\hline & PARATEBUENO (CUND.) & 258.292 & $45,3 \%$ \\
\hline & GUAVIARE & & \\
\hline & META & & \\
\hline \multirow{3}{*}{ SANTANDERES } & NORTE DE SANTAND & & $7.1 \%$ \\
\hline & SANTANDER & 40.568 & \\
\hline & TOTAL & 570.802 & $100 \%$ \\
\hline
\end{tabular}

Fuente: DANE (2016). Censo Nacional Arrocero. Recuperado de https://www.dane.gov.co/files/investigaciones/agropecuario/censonacional-arrocero/presentacion-4to-censo-nacional-arrocero-2016.pdf

Las razones que generan presión de poder inteligente en el mercado local son el conflicto armado en los territorios campesinos arroceros, las condiciones de desigualdad y el empobrecimiento de la población. Si analizamos ese poder a la luz de la propuesta de Nye (2011), existen tres aristas, el poder duro (hard power) basado en los recursos militares y económicos [ejercido por los grupos armados en el territorio]; poder suave (soft power) basado en instituciones, cultura y legitimidad de las acciones [crecimiento económico es igual a desarrollo] y poder inteligente (smart power), integración de diplomacia, defensa, desarrollo [la implementación de un TLC que incida en el sector productor arrocero] (Nye, 2011).

Los autores Augelli y Murphy, (1988); Cox, (1987); Cox y Sinclair (1996); Davies (1999); Gill (1990), Neufeld, Hoffman, Linklater y Ahsley (1993), Mohanty (1991), Escobar (2007) y Bieler (2008) sostienen, desde perspectivas distintas, que "la idea del crecimiento económico no llega al desarrollo humano, entendido en el sentido general de mejoramiento en las condiciones de vida o el 'estar bien', [siendo unos aspectos materiales y espirituales que permiten al ser humano estar bien según José Martínez de Sousa]" (Colmenares, 2013). Así mismo, hay quienes sostienen que el desarrollo económico tiene que ampliar la noción de desarrollo a un campo más humano, que vincule el bienestar del individuo y la libertad económica (Soubbotina \& Sheram, 2000).

Esa libertad económica y el bienestar para los productores surgió de la iniciativa del movimiento social campesino arrocero denominado 'Dignidad Arrocera', que se legitimó durante el Paro Agrario de 2013, según un líder sindical.

Un día en una asamblea que teníamos con los de la organización dijeron - esto es un problema de dignidad, no nos podemos dejar joder-. Y bueno, pues nos pareció bonito el nombre, entonces pongámosle Dignidad Arrocera y empezamos a afirmarnos como Dignidad. Y de ahí es que salieron lo que popularmente llama el gobierno como 'Dignidades' que es una representativa de los gremios (entrevista \# 1, 3 de febrero del 2015). 
Este movimiento social campesino dio la posibilidad de unir a los trabajadores del gremio arrocero del Huila, Tolima, Casanare, Córdoba y Santander. La posición de 'Dignidad Arrocera' es demandar los derechos sociales y económicos como posibilidad de renegociar el TLC, para que sea más equitativo y cumpla con normativas de la OMC. Un objetivo es recuperar las semillas nativas para contrarrestar esa política y que el campesino no dependa de las semillas certificadas. Esa plataforma gremial dio paso a la creación de otras dignidades como la Papera, Cebollera, entre otras. Esas organizaciones facilitaron la construcción de la 'Mesa Agraria Nacional'.

Con ayuda de la coordinación de Fensuagro y de otros procesos como los indígenas, se ha conformado la Cumbre de los Pueblos [la Mesa Única de Interlocución y Participación -NUN- (consolidada hasta el 2014) con la idea de mejorar las condiciones comerciales y bienestar de los pequeños y medianos productores]. Se vienen negociando con el gobierno ocho puntos fundamentales que tienen que ver con la problemática de Colombia [...]: la paz, zonas de reserva campesina -ZRC-, la salida de cultivos ilícitos, la problemática minero-energética que es causa del despojo de la tierra y la renegociación del TLC (entrevista \#3, 17 de febrero 2015).

Este movimiento, con ayuda de la Dignidad Arrocera, crearon una red de comercio campesino denominado 'de campesino a campesino' o Programa de Agricultura Familiar que consiste en una red de comercialización, que establece espacios para el trueque y comercialización [sic], es una tarea de desentierro y rescate del viejo paradigma de las sociedades agrarias que buscan promover la seguridad alimentaria de sus comunidades, a través del diseño de auténticos modelos de empresas familiares rurales en pro del respeto a la naturaleza (Fensuagro, 2013).

\section{CONCLUSIONES}

El crecimiento económico con el TLC en Colombia es una manera de favorecer a algunos sectores y afectando a otros. En el caso del sector productor arrocero, las afectaciones son la desventaja comparativa en términos comerciales, desventajas en las relaciones de intercambio y el desinterés de políticas gubernamentales estadounidenses hacia el mercado arrocero colombiano, pérdida del territorio a causa de la deuda y el conflicto armado, la falta de tecnificación sanitaria y fitosanitaria, aumento desmesurado del contrabando, oligopolio de las molineras y carencia de divulgación sobre las investigaciones de agroquímicos naturales. Estas condiciones han creado un escenario desfavorable para el sector productor arrocero, debido a las relaciones de poder y la importancia de la inversión de EEUU para el Gobierno Nacional.

La inconformidad de los sectores agrícolas posibilitó la conformación de una mesa nacional agraria que trabaja en pro de los derechos campesinos, lucha contra los obstáculos de comercialización a los pequeños productores. El movimiento social arrocero, mediante sus prácticas populares de producción y comercialización ha vinculado agricultores, investigadores y científicos que apoyan el proceso de crecimiento y desarrollo del agro. Tarea que sigue sin concretarse. Por tanto, se recomienda que busquen mejorar las condiciones de intercambio, aumento de la soberanía en el territorio y mecanismos de renegociación del TLC.

\section{REFERENCIAS BIBLIOGRÁFICAS}

Acosta, A. (2006). TLC: Entre la verdad y la mentira . En F. F. Alberto Acosta, El rostro oculto del TLC (págs. 31-114). Quito: Docutech.

Canal Llanero. (2015). Continúa crisis arrocera en 2015. Recuperado de https://www.youtube.com/watch?v $=$ pqf35Ciqkjo\&list $=$ WL\&index $=89$.

Armitage, J. N. (2007). A smarter, more secure America. Washington: CSIS Commission On Smart Power.

Balassa, B. A. (1965). Trade liberalization and revealed comparative advantage. Manchester: The Manchester School of Economic and Societal Studies.

Bieler, A. y. (2008). Social Forces in the Making of New Europe . En A. y. Bieler, The Restructuring of European Social Relations in the Global Political Economy (pág. 264). Nova Scotia.

CINEP. (2014). Desde el corazón del movimiento campesino. Bogotá: Gente Nueva. 
Colmenares, J. S. (2013). Nuevo modo de desarrollo: una utopía posible. Bogotá: Editorial Aurora .

Cox, R. (2002). Power and Knowledge: Toward a new ontology of world order" En The Political Economy of a Plural World: Critical Reflections on Power, Morals and Civilizations. London: Roultedge.

Cox, R. (2013). Fuerzas sociales, estados y órdenes Mundiales: Más allá de la Teoría de Relaciones Internacionales. Grupo de Estudios de Relaciones Internacionales (GERI) - UAM 24,129-160.

DNP (2017). Análisis del comercio internacional colombiano y diversificación de exportaciones (2002-2015): Canadá, Estados Unidos y Unión Europea . Bogotá.

Escobar, A. (2007). La invención del tercer Mundo - Construcción y deconstrucción del desarrollo. En Visualización de una era post-desarrollo (pp. 355-379). Caracas: Fundación Editorial El perro y la rana.

Federación de Desarrollo -Fedesarrollo-. (2007). Impacto económico del TLC Con Estados Unidos en la región Bogotá-Cundinamarca . Bogotá: Cámara y Comercio de Bogotá .

Federación Nacional de Arroceros -Fedearroz-. (2011). Dinámica del sector arrocero de los llanos orientales de Colombia. Arroz, 6-127.

Federación Nacional Sindical Unitaria Agripecuaria-Fensuagro- (2013). Modelos de producción de los sistemas agrícolas (campesino, indígena y afro). Bogotá: CUT.

Finagro. (2015.). Fuentes de financiamiento agropecuario. Recuperado https://www.finagro.com.co/ productos-y-servicios/pran.

Fukuyama, F. (1992). The End of History and the Last Man. New York: Simon \& Schuster.

Hart, J. (1976 ). Dominance In International Politics, International Organization.

Krugman, P. y. (2000). International Economics: Theory and Policy.New York: Addison- Wesley.

Leontief, W. (1985). Análisis input-output. Barcelona: Orbis.

Lewis, A. (1954). Economic Development with Unlimited Supplies of Labour. Manchester: University of Manchester.

Ministerio de Agricultura y Desarrollo Rural (2011). Boletín De Análisis Por Producto. Documento de trabajo, Dirección de Política Sectorial-Grupo de Análisis Sectorial del Meta.

Ministerio de Industria y Turismo (2014). Acuerdo de Promoción Comercial entre la República de Colombia y Estados Unidos de América. Recuperado de http://www.tlc.gov.co/publicaciones.php?id=14853.

Mohanty, C. (1991). Cartographies of struggle, third world women ando the politics of feminism . En A. R. Chandra Mohanty, Third world women and the politics of feminism (pp.1-47). Bloomington: Indiana University press.

North, D. (2002). Institutions and Economic Growth: A historical Introduction World Development.

Nye, J. (2011). The Future of Power. New York: Public Affairs.

OXFAM. (2018). Cinco datos escandalosos sobre la desigualdad extrema global y cómo combatirla. Recuperado de https://www.oxfam.org/es/iguales/cinco-datos-escandalosos-sobre-la-desigualdadextrema-global-y-como-combatirla

Organización de los Estados Americanos -OEA- .(2014). Sistema de Información sobre Comercio Exterior entre Colombia y Estados Unidos. Recuperado de http://www.sice.oas.org/ctyindex/COL/COLagreements_s. asp.

Organización Mundial de Comercio. (2018). Informe sobre Acuerdos comerciales regionales. Ginebra. Recuperado de OMC: https://www.wto.org/spanish/tratop_s/region_s/regfac_s.htm

Procolombia (2012). "Tratado de libre comercio entre Colombia y Estados Unidos: más comercio, más empleo". Recuperado de .http://www.bancoldex.com/documentos/3758_TLC_eltiempo_03.pdf

Keohane, R. (1977). Poder e interdependencia: la política mundial en transición. Buenos Aires: Grupo editoral latinoaméricano SRL.

Rodriguez, F. (1999). Trade policy and economic growth: a skeptic's guide to the cross-national evidence . En F. a. Rodriguez. National Bureau of Economic Research.

Rostow, W. (1960). The stages of economic growth, a Non-Communist Manifesto. Cambridge: Cambridge University Press.

Scott, L. \& Vollrath, T. (1992). Global competitive advantages and overall bilateral complementary in agriculture. USDA/IRS Statistical, (pp.745-850).

Sectorial, P. (2014). La Historia del Comercio Exterior en Colombia. Recuperado de https://www.sectorial. co/index.php?option=com_content\&view=article\&id=302: Ia-historia-del-comercio-exterior-encolombia\&cati 
Soubbotina, T. P., \& Sheram, K. A. (2000). Beyond Economic Growth : Meeting the Challenges of Global Development. WBI Learning Resources. Washington, DC: World Bank.

Entrevista \#1, 03 de febrero 2015, departamento del Meta.

Entrevista \#2, 04 de febrero 2015, Bogotá.

Entrevista \#3, 11 de febrero 2015, departamento del Meta.

Entrevista \#4, 17 de febrero 2015, departamento del Meta.

Entrevista \#5, 21 de febrero 2015, Bogotá. 